\title{
Interleukin-8 as a Biomarker for Disease Prognosis of Coronavirus Disease-2019 Patients
}

OPEN ACCESS

Edited by:

Ruchi Tiwari,

U.P. Pandit Deen Dayal Upadhyaya

Veterinary University, India

Reviewed by:

Muhammad Bilal,

Shanghai Jiao Tong University, China

Ranjan K. Mohapatra,

Government College of Engineering,

Keonjhar, India

*Correspondence:

Heng Yang

yhmyt@hotmail.com

Genhong Cheng

gcheng@mednet.ucla.edu

${ }^{\text {t}}$ These authors share first authorship

${ }^{\ddagger}$ These authors share senior authorship

Specialty section:

This article was submitted to

Viral Immunology,

a section of the journal

Frontiers in Immunology

Received: 03 September 2020

Accepted: 26 November 2020

Published: 08 January 2021

Citation:

Li L, Li J, Gao M, Fan H, Wang Y, Xu X,

Chen C, Liu J, Kim J, Aliyari R,

Zhang J, Jin Y, Li X, Ma F, Shi M,

Cheng $G$ and Yang $H$ (2021)

Interleukin-8 as a Biomarker for

Disease Prognosis of Coronavirus

Disease-2019 Patients.

Front. Immunol. 11:602395. doi: 10.3389/fimmu.2020.602395
Lili $\mathrm{Li}^{1,2 \dagger}$, Jie $\mathrm{Li}^{3 \dagger}$, Meiling Gao ${ }^{1,2,3 t}$, Huimin Fan ${ }^{1,2 \dagger}$, Yanan Wang ${ }^{4 \dagger}$, Xin $\mathrm{Xu}^{1,2}$, Chunfeng Chen ${ }^{4}$, Junxiao Liu ${ }^{1,2}$, Jocelyn Kim ${ }^{5}$, Roghiyh Aliyari ${ }^{5}$, Jicai Zhang ${ }^{3}$, Yujie Jin ${ }^{3}$, Xiaorong $\mathrm{Li}^{1,2}$, Feng $\mathrm{Ma}^{1,2}$, Minxin Shi ${ }^{6}$, Genhong Cheng ${ }^{{ }^{*}}$ and Heng Yang ${ }^{1,2^{\star} \neq}$

1 Center for Systems Medicine, Institute of Basic Medical Sciences, Chinese Academy of Medical Sciences \& Peking Union Medical College, Beijing, China, ${ }^{2}$ Cheng Lab, Suzhou Institute of Systems Medicine, Suzhou, China, ${ }^{3}$ Department of Laboratory Medicine, TaiHe Hospital, Hubei University of Medicine, Shiyan, China, ${ }^{4}$ Department of Scientific Research, Suzhou Func Biotech Inc, Suzhou, China, ${ }^{5}$ Department of Microbiology, Immunology \& Molecular Genetics, University of California, Los Angeles, Los Angeles, CA, United States, ${ }^{6}$ Department of Surgery, Affiliated Tumor Hospital of Nantong University, Nantong, China

The widespread prevalence of coronavirus disease-2019 (COVID-19) which is caused by severe respiratory syndrome coronavirus 2 (SARS-CoV-2) infection, has resulted in a severe global public health emergency. However, there are no sensitive biomarkers to predict the disease prognosis of COVID-19 patients. Here, we have identified interleukin-8 (IL-8) as a biomarker candidate to predict different disease severity and prognosis of COVID-19 patients. While serum IL-6 become obviously elevated in severe COVID-19 patients, serum IL-8 was easily detectible in COVID-19 patients with mild syndromes. Furthermore, IL-8 levels correlated better than IL-6 levels with the overall clinical disease scores at different stages of the same COVID-19 patients. Thus, our studies suggest that IL-6 and IL-8 can be respectively used as biomarkers for severe COVID-19 patients and for COVID-19 disease prognosis.

Keywords: cytokine serum profile, biomarker, coronavirus disease-2019 prognosis, respiratory syndrome coronavirus 2, cytokine storm, interleukin-6, interleukin-8

\section{INTRODUCTION}

Since the initial outbreak in December of 2019, severe respiratory syndrome coronavirus 2 (SARSCoV-2) infection and its associated coronavirus disease-2019 (COVID-19) has quickly developed into a global epidemic (1-3), causing over 70 million confirmed COVID-19 cases and 1.6 million associated deaths $(2,4-6)$. SARS-CoV-2 is a novel betacoronavirus belonging to the large coronavirus family. The coronavirus family includes Alphacoronavirus [i.e. Human coronavirus 229E, Human coronavirus NL63), Betacoronavirus (SARS-CoV, SARS-CoV-2, Middle East respiratory syndrome (MERS), Human coronavirus OC43), Gammacoronavirus (Avian coronavirus (IBV)], and Deltacoronavirus [Bulbul coronavirus HKU11 (Bulbul-CoV HKU11)] $(7,8)$. In the past two decades, two large scale pandemics have been caused by coronavirus, SARS in 2003 and MERS in $2012(9,10)$. SARS-CoV-2 infection was firstly reported at the end of 2019 and rapidly spread across the world in a few months, posing a major threat to the health of people around the world. 
Symptoms of COVID-19 include fever, cough, shortness of breath, tissue damage, multiorgan failure, and death. The outcomes of COVID-19 vary from mild symptoms to acute resolved or fatal pneumonia. Most COVID-19 patients were classified as mild (81\%), $14 \%$ as severe, and $5 \%$ as critical. The overall case-fatality rate (CFR) was $2.3 \%$ and all the deaths were from critical patients who represented about almost half of critical cases (11). Elderly patients and those with preexisting co-morbid conditions have higher risks to develop critical illness and die from the COVID-19 disease $(11,12)$.

Diagnosis for COVID-19 patients includes two main methods, nucleic acid testing for detection of SARS-CoV-2 RNA and serological assay for detection of specific IgG and IgM antibodies against SARS-CoV-2 (13). To avoid the false negative rate of reverse transcriptase polymerase chain reaction (RT-PCR) test and improve the accuracy of diagnosis, researchers have suggested a strategy of combining RT-PCR test, antibody test, and CT scan for COVID-19 diagnosis (1416). Meanwhile, some researchers also tried to find predictors of COVID-19 disease prognosis. Although the viral load, viremia, and RNA in sera of SARS-CoV-2 correlated with the severe outcome in COVID-19 patients (17-20), sensitive biomarkers representing the prognosis of COVID-19 patients are stilled lacked.

SARS-CoV-2 infection induces exuberant inflammatory responses and increased secretion of interleukin-1 $\beta$ (IL-1 $\beta$ ), interferon- $\gamma($ IFN- $\gamma)$, interferon inducible protein 10 (IP-10), monocyte chemotactic protein 1 (MCP-1), interleukin-4 (IL-4), and IL-10. In addition, intensive care unit (ICU) patients with more severe disease process have higher plasma levels of IL-2, IL7, IL-10, granulocyte colony stimulating factor (GCSF), IP-10, MCP-1, macrophage inflammatory protein-1a (MIP-1A), and tumor necrosis factor- $\alpha$ (TNF- $\alpha)$ than non-ICU patients $(21-$ 24). Among severe COVID-19 cases, the majority of the patients with respiratory distress syndrome were associated with high systemic IL- $1 \beta$, TNF- $\alpha$, and IL-6 levels $(25,26)$. These reports suggest a possible connection between proinflammatory cytokine induction and adverse effects of COVID-19. IL-6 is considered a relevant indicator in predicting severe course of COVID-19 disease and target for COVID-19 treatment (27-31). Besides inflammatory cytokines, some other predictors, such as lymphocyte number, lactate dehydrogenase, D-dimer, and IL2R have also been identified as out of normal range in COVID-19 patients $(12,32,33)$. Nevertheless, available biomarkers for predicting the disease progression of COVID-19 patients are still limited.

In this study, we characterized the serum profiles of 40 cytokines in COVID-19 patients at different disease stages. While IL-6 was an available indicator in severe COVID-19 patients, IL- 8 performed better in indicating the progress of COVID-19 disease status from mild to severe. IL-8 plasma levels were elevated in both mild and severe COVID-19 patients and increased with disease progression. Our work showed that IL-6 and IL-8 could be combined used as biomarker for different disease stages of COVID-19 patients.

\section{MATERIALS AND METHODS}

\section{Quantibody Human Inflammation Array Assay}

Forty human cytokines of 8 COVID-19 patients' sera were evaluated using Quantibody Human Inflammation Array-3 (RayBiotech, Inc.) according to the manufacturer's protocol. After incubated with 8 COVID-19 patients' sera and 8 standard samples, the slides were scanned using a laser scanner (Axon GenePix; Molecular Devices) and images were collected with GenePix Pro 6.1.0.4 software (Molecular Devices). Data from the images were further gathered using GenePix Array list files (RayBiotech, Inc.) and converted to concentration with RayBio Q Analyzer software (RayBiotech). The comparative concentrations of the 40 cytokines were displayed with heatmap as indicated in Figure 1B.

\section{Clinical Investigations and Data Analysis}

Clinical investigations in COVID-19 patients and healthy volunteers were approved by the Ethics Committee of Taihe hospital (2020KS031). Sera from human donors were collected after the patients were admitted to the hospital with obvious SARS-CoV-2 infection symptoms like fever, radio-graphic evidence of pneumonia, low or normal white-cell count or low lymphocyte count and positive SARS-CoV-2 nucleic acid testing. All the sera were collected under a protocol approved by the local governing human research protection committee and used under the approvement of Taihe hospital. We used a modified Acute Physiology and Chronic Health Evaluation (APACHE II) scoring system to evaluate the disease severity of 5 patients during the hospitalization. Briefly, the APACHE II score of each patient was evaluated based on 12 physiologic measurements (Body temperature, mean arterial pressure, heart rate, respiratory rate, alveolar-arterial oxygen (A-a) gradient; if fractional inspired oxygen concentration is $\geq 0.5$, arterial oxygen tension $\left(\mathrm{PaO}^{2}\right)$; if fractional inspired oxygen concentration is $<0.5$, serum bicarbonate $\left(\mathrm{HCO}_{3}\right)$; if there is no arterial blood gas analysis, arterial $\mathrm{pH}$, serum sodium, serum potassium, creatinine, hematocrit, white blood cell count, Glasgow Coma Scale score), age of the subject and comorbid conditions. Physiologic signs and laboratory values used to calculate the APACHE II score must have been obtained using the worst physiologic variable within the $24 \mathrm{~h}$ period. A higher score is associated with more severe disease and a higher risk of hospital death.

\section{Enzyme-Linked ImmunoSorbent Assay}

IL-6 and IL-8 levels in COVID-19 patients and healthy people's sera were measured with Human IL-6 ELISA Set (BD biosciences) and Human IL-8 ELISA Set (BD Biosciences) according to the manufacturer's protocol. Briefly, the plates were coated with the capture antibodies at $4^{\circ} \mathrm{C}$ for overnight and then washed with 200 $\mu \mathrm{l} /$ well 1xPBST buffer (1xPBS supplemented with $0.05 \%$ Tween20) for 3 times. These coated plates were blocked with $100 \mu \mathrm{l} /$ well blocking solution ( $1 \% \mathrm{BSA}$ in PBS buffer) at $37^{\circ} \mathrm{C}$ for $1 \mathrm{~h}$. After washing three times, $100 \mu \mathrm{l} /$ well diluted sera were added into the plates and incubated at $37^{\circ} \mathrm{C}$ for $2 \mathrm{~h}$, followed by washing for three times. Diluted capture antibodies at $100 \mu \mathrm{l} /$ well were then added 
A

\begin{tabular}{ccccc}
\hline Patient & $\begin{array}{c}\text { Clinical } \\
\text { classification }\end{array}$ & Sample & $\begin{array}{c}\text { Nucleic acid } \\
\text { test results }\end{array}$ & $\begin{array}{c}\text { Nucleic acid } \\
\text { detection } \\
\text { site }\end{array}$ \\
\hline Patient 1\# & Severe & Throat Swab & + & ORF 1ab \\
& Recovered & Throat Swab & - & ORF 1ab \\
Patient 2\# & Severe & BALF & + & ORF 1ab \\
& Recovered & Whole Blood & - & ORF 1ab \\
Patient 3\# & Severe & Throat Swab & + & ORF 1ab \\
& Recovered & Throat Swab & - & ORF 1ab \\
Patient 4\# & Death & Phlegm & + & ORF 1ab \\
\hline
\end{tabular}

B

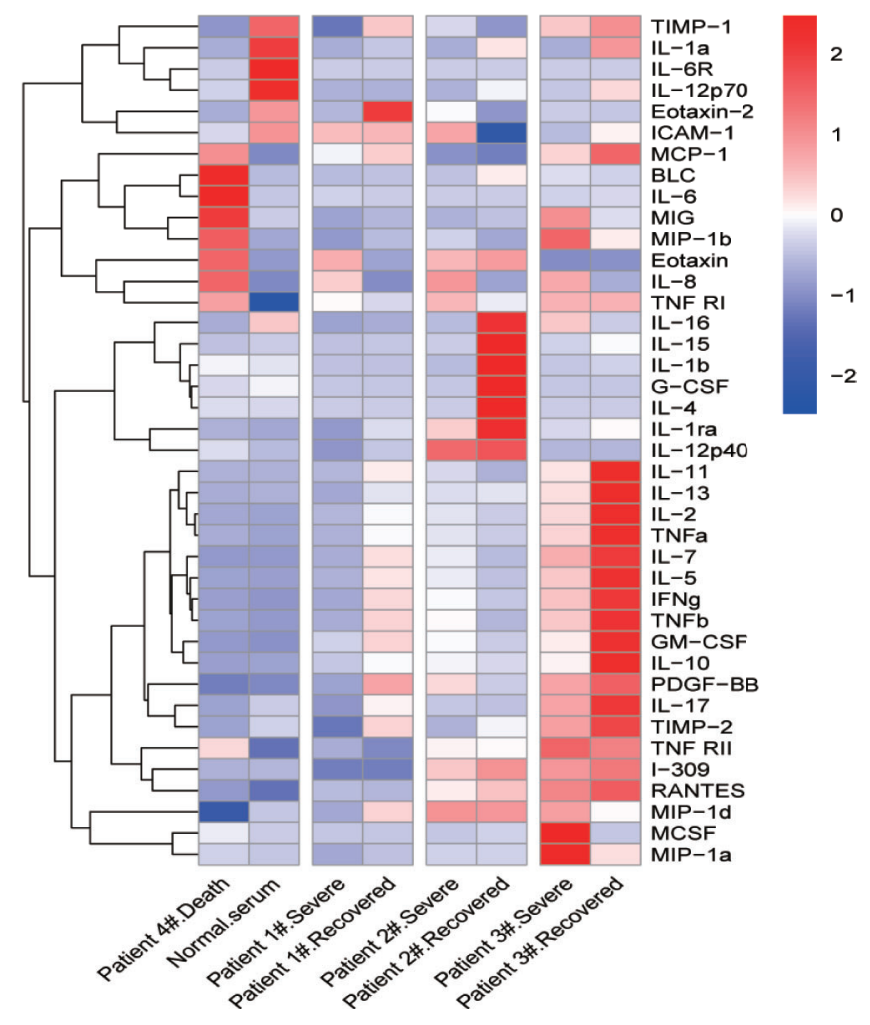

FIGURE 1 | Multiple cytokine profile from different clinical outcomes of SARS-CoV-2 infected patients. (A) Clinical outcomes and nucleic acid test results of four SARS-CoV-2 infected patients. The patients were divided into two groups by clinical outcomes: severe and recovered. Patient 4\# died in the hospital. (B) Heatmap of cytokines in sera from COVID-19 patients during different clinical stages. Because of the death, we did not get recovered serum from patient $4 \#$. We then added normal serum as a control. Cytokine levels were detected using Quantibody ${ }^{\circledR}$ Human Inflammation Array-3.

into the plates and incubated at $37^{\circ} \mathrm{C}$ for $1 \mathrm{~h}$, followed by washing for three times. Diluted secondary antibody was added at $100 \mu \mathrm{l} /$ well into the plates and incubated at $37^{\circ} \mathrm{C}$ for $1 \mathrm{~h}$, followed by washing for five times. As the final step of ELISA, 100 $\mu \mathrm{l} /$ well 3,3',5,5'-Tetramethylbenzidine (TMB) was added into the plate and incubated for 5-10 min with light protection and the reaction was terminated by addition of $50 \mu \mathrm{l} /$ well $2 \mathrm{M} \mathrm{H}_{2} \mathrm{SO}_{4}$. The absorbance at $450 \mathrm{~nm}$ in individual wells were measured by SpectraMax ${ }^{\circledR}$ i3 (Molecular Devices) plate reader.

\section{Statistics}

Statistical analyses were performed with GraphPad Prism 7 software and $\mathrm{R}$ Studio version 3.6.3. The continuous variables were presented as mean \pm SD. Data with normal distribution were analyzed by one-way ANOVA or unpaired two-tailed Student's $t$ tests, and $\mathrm{P}$ values were indicated by ns, not significant, ${ }^{\star} P<0.05$, ${ }^{\star *} P<$ $0.01,{ }^{* *} P<0.001$, and ${ }^{* * * *} P<0.0001$.

\section{RESULTS}

\section{Multiple Cytokine Profile From Different Clinical Outcomes of SARS-CoV-2 Infected Patients}

Cytokine storm has been found to be a part of COVID-19 associated severe diseases. To identify differential cytokine profiles potentially associated with disease status of COVID-19 
patients, we tested 40 human inflammatory related cytokines in the sera of 4 hospitalized patients with confirmed COVID-19 by the Quantibody Human Inflammation Array-3 (Figure 1A). The patients were classified as "severe" during their critical illness or "recovered" based on the conversion to negative nucleic acid test results. Serum samples were collected from these patients at their severe and recovered status, except for patient 4\# who was dead from COVID-19 and one serum sample was obtained before the death of the patient (Patient $4 \#$ death). We also randomly select a serum sample from a healthy individual (Normal serum). The array result showed strong variations in the cytokine levels among different patients and at different stages for the same patients. Despite these variations, the levels of IL-8 were apparently elevated at the severe stage of all four patients, when compared with recovered and healthy people. Interestingly, the change in IL-6 levels was not so obvious except in patient 4\# (Figure 1B).

\section{Cytokine Levels in COVID-19 Severe and Recovered Patients' Sera}

Besides IL-6 and IL-8 levels, other inflammatory cytokines like Blymphocyte chemoattractant 1 (BLC1, also called CXCL13), monokine induced by IFN-gamma (MIG, also called CXCL9), macrophage inflammatory protein 1 beta (MIP-1b), eotaxin-1 (also called CCL11), tumor necrosis factor-receptor 1 (TNF-R1), and MCP-1 also appeared at very high levels in the patient \# 4 compared to the normal serum (Figures 2A-H). These cytokines could potentially be indicators of disease severity. Interestingly, the IL-8 levels showed a distinct difference between severe and recovered stages (Figure 2B). These results suggest that the level of IL- 8 is better than IL- 6 as an indicator for COVID-19 disease status.

\section{IL-8 Performed Better in Distinguishing COVID-19 Patients From Healthy People}

To verify IL-8 as an indicator of COVID-19 disease status, we measured IL- 6 and IL- 8 concentrations in the sera of 138 COVID-19 patients and 26 healthy people by ELISA assay. Compared to healthy people, both IL- 8 and IL-6 levels in the sera of COVID-19 patients were significant higher (Figures 3A, B). Receiver operating characteristic (ROC) analysis showed that IL-8 had a higher area under the curve (AUC) (0.9776) than IL-6 (0.8417) (Figures 3C, D). The results indicate that IL-8 performs better in distinguishing COVID-19 patients from healthy people.

\section{While IL-6 Represents for COVID-19 Patients With Severe Disease Conditions, IL-8 Is a Better Indicator of Overall COVID-19 Disease Status}

For the prediction of COVID-19 patient's disease status, we analyzed IL-6 and IL-8 levels among healthy people, mild, and severe COVID-19 patients. The serum levels of IL- 8 were higher in COVID-19 patients with mild symptoms than in healthy people and further elevated in severe COVID-19 patients. However, the serum levels of IL-6 were increased obviously only in severe COVID-19 patients and showed no statistically significant differences between COVID-19 patients with mild symptoms and healthy people (Figures 4A-D). These data suggest that while IL-6 represents for COVID-19 patients with severe disease conditions, IL- 8 is a better indicator of overall COVID-19 disease status.

\section{IL-8 May Serve as a Biomarker to Indicate the COVID-19 Disease Prognosis}

To compare IL- 6 versus IL- 8 for the disease prognosis prediction, we monitored the changes of IL- 6 and IL- 8 concentrations in the disease progression offive COVID-19 patients with different clinical scores, which represent a combination of multiple physiologic measurements associated with the COVID-19 diseases. The levels of IL-8 seemed to correlate well with different patient's clinical scores at multiple time points (Figures 5A-E). On the other hand, the levels of IL- 6 remained low at most of the time points except patients at very high clinical scores. Our studies therefore indicate that IL-8 may serve as a biomarker to indicate the COVID-19 disease prognosis.

\section{DISCUSSION}

Since the outbreak of SARS-CoV-2 infection, the global pandemic of COVID-19 has caused about a million deaths among over fifty million confirmed cases worldwide (34-36). Although PCR-based detection of SARS-CoV-2 viral RNA and ELISA-based detection of antibodies against SARS-CoV-2 viral proteins have been developed for the diagnosis of infected versus uninfected patients $(37,38)$, there is urgent need to identify biomarkers for the disease prognosis of COVID-19 patients. In the present studies, we have provided evidence that while IL- 6 may be used for the diagnosis of severe COVID-19 patients, IL-8 is overall a better biomarker for the COVID-19 disease prognosis.

SARS-CoV-2 infection caused a wild range of symptoms among different COVID-19 patients. The outcomes of COVID-19 patients also varied from recovery to death. While young and healthy COVID-19 patients may be asymptomatic or have mild symptoms, elderly patients and those with preexisting health conditions tend to develop severe and fatal disease after infection with SARS-CoV-2 $(5,39)$. Because of the wide and variable range of clinical symptoms and progression, it is difficult to predict the disease progress and outcome for individual COVID-19 patients. Through comparing the cytokine levels in the serum samples of COVID-19 patients at longitudinal timepoints during severe illness or at recovery, we have identified differential cytokine profiles potentially associated with COVID-19 disease status. Besides IL-6, which has been reported as a potential biomarker for COVID-19 patients $(28,40)$, we found that both IL- 6 and IL-8 serum levels were elevated in COVID-19 patients with severe diseases. In particular, we found that serum levels of IL-6 and IL8 were significantly higher in COVID-19 patients as compared with heath donors with the AUC, which represents the combination of detecting sensitivity and specificity, at 0.84 and 0.98 , respectively. 
A

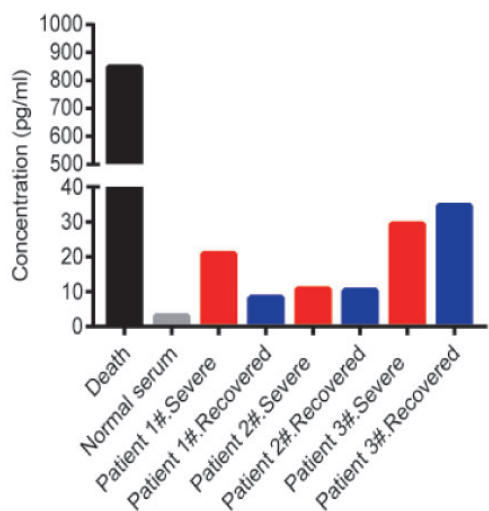

C

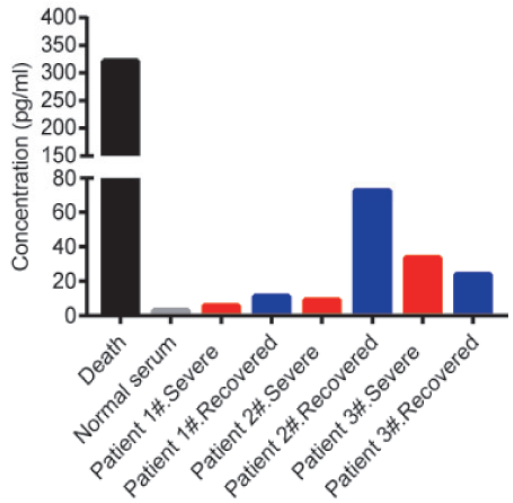

F

Eotaxin-1

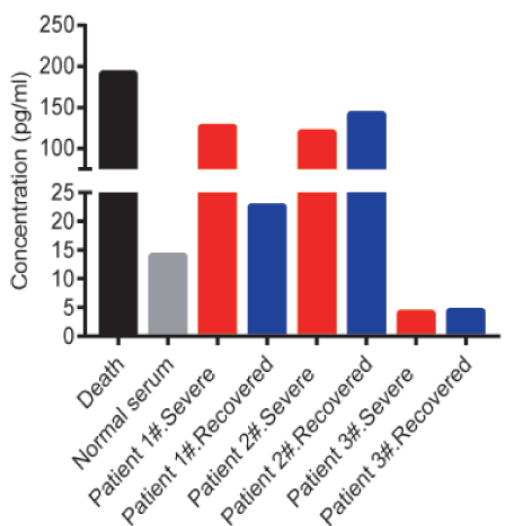

B

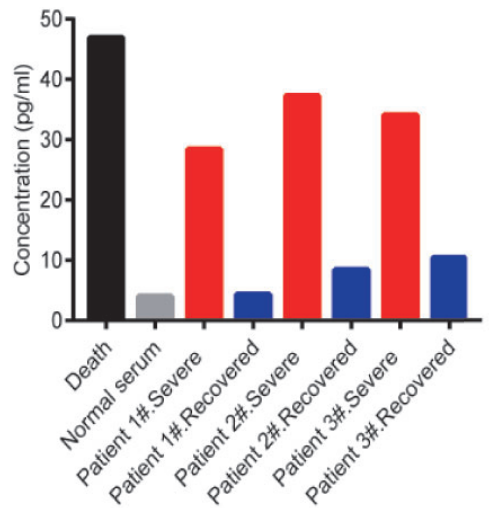

D

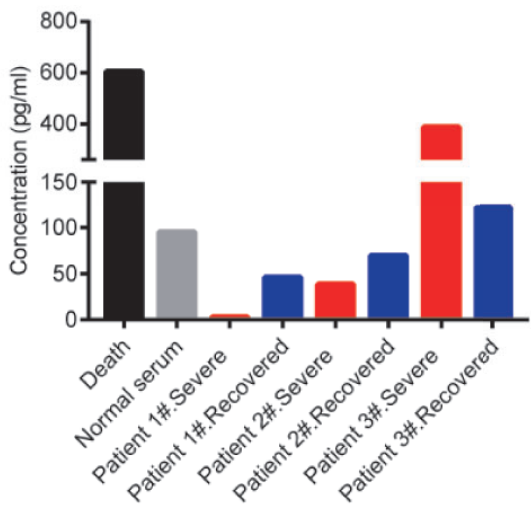

G

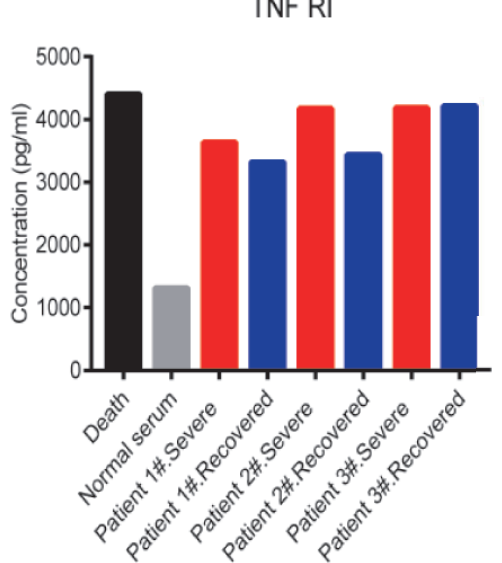

Death

Normal serum

Severe

Recovered
E

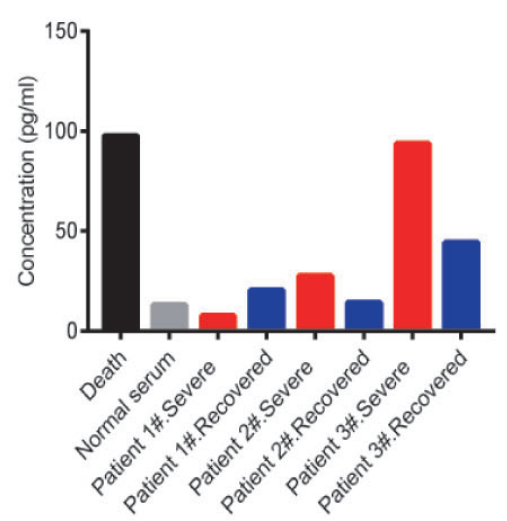

H

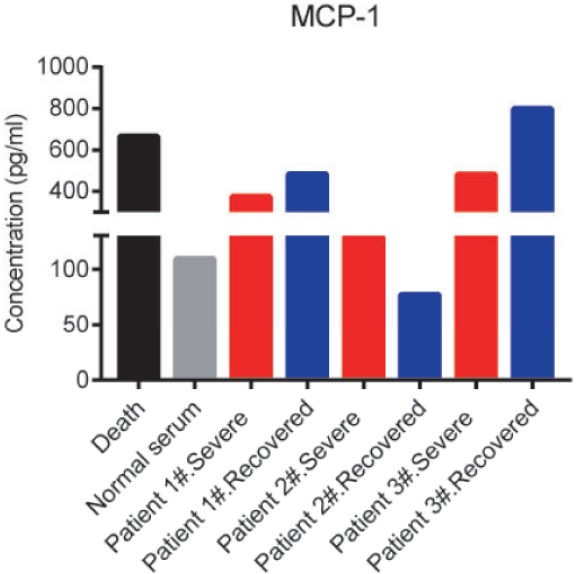

FIGURE 2 | Cytokine levels in COVID-19 severe and recovered patients' sera. Cytokine levels of IL-6 (A), IL-8 (B), BLC (C), MIG (D), MIP-1b (E), Eotaxin-1 (F), TNF RI (G), and MCP-1(H) in COVID-19 severe and recovered patients' sera. Every column represents one serum sample of COVID-19 patients. 
A

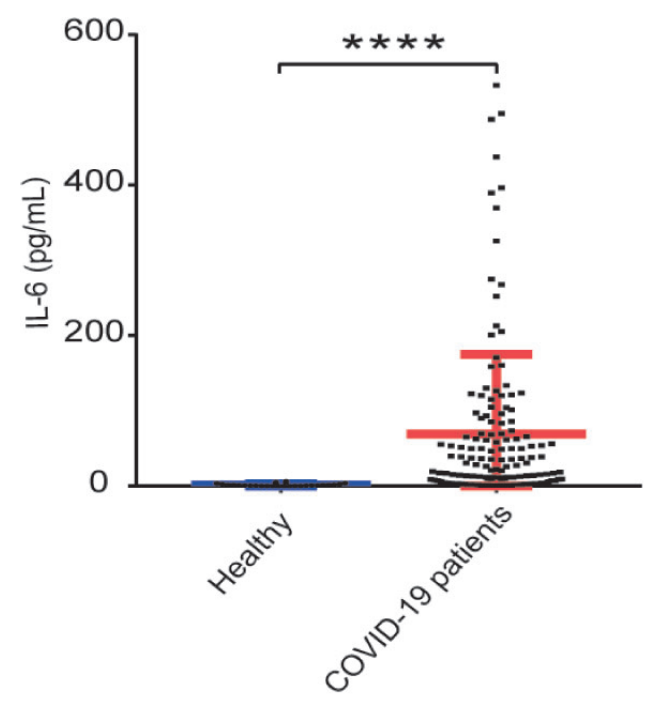

C

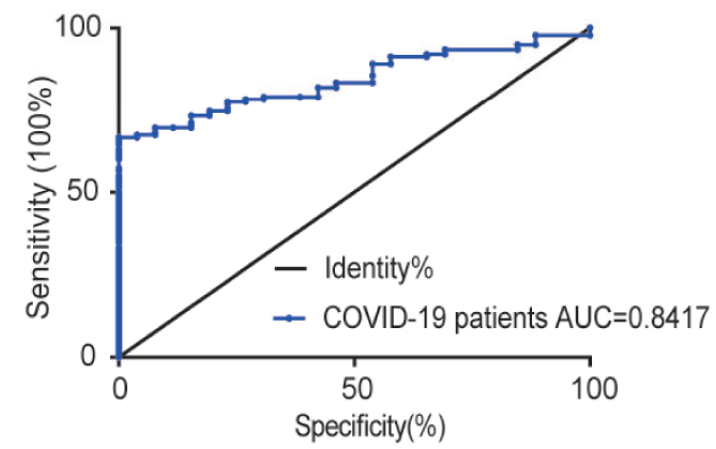

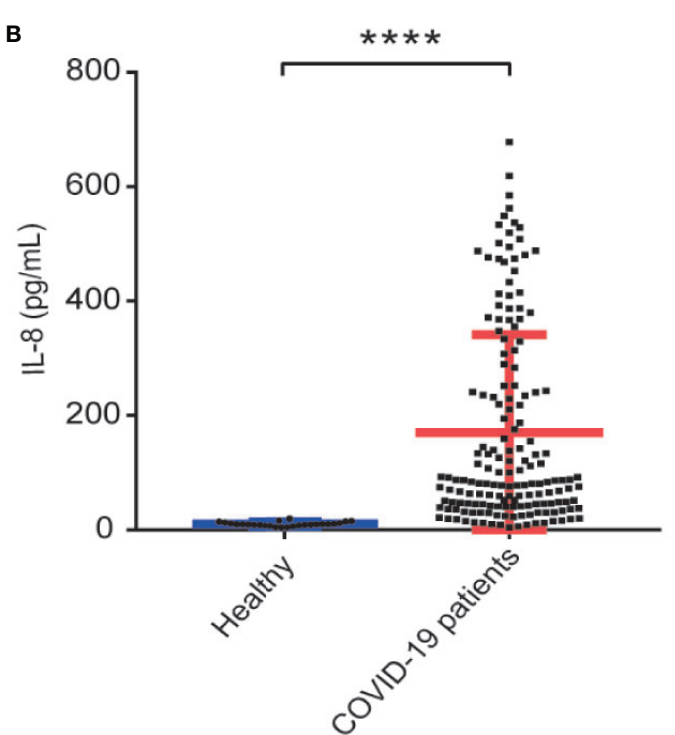

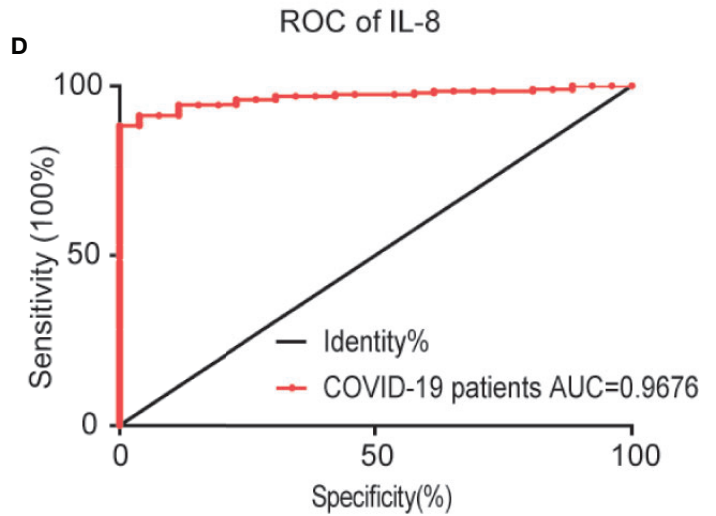

FIGURE 3 | IL-8 performed better in distinguishing COVID-19 patients from healthy people. (A, B) Scatter plot of IL-6 (A) and IL-8 (B) levels detected by ELISA in healthy and COVID-19 patients' sera. (C, D) The detectability performance of IL-6 (C) and IL-8 (D) in COVID-19 patients was estimated using ROC curve analysis and compared with the AUC. ELISA data (A, B) are shown as Mean \pm SEM, ${ }^{\star \star \star \star *} p<0.0001$, unpaired Student $t$ test.

More importantly, our studies indicated that IL-6 and IL-8 may be differentially used as biomarkers for the severity and prognosis of the COVID-19 associated diseases. The levels of IL- 6 were low in most of the COVID-19 patients with mild symptoms and were elevated in patients with severe symptoms, which therefore may be used as a biomarker for severe COVID-19 patients $(25,41)$. On the other hand, the serum levels of IL-8 were remarkably higher in COVID-19 patients with either mild or severe diseases as compared with the healthy donors. Furthermore, the IL- 8 levels correlated better than IL-6 with the overall clinical disease scores at the different time points in the same COVID-19 patients, which can therefore be used as a disease prognosis biomarker.

Cytokine storms have been reported as a part of the COVID-19 associated severe diseases $(24,42,43)$. Blocking antibodies or small molecules targeting IL- 6 or its signal transduction pathways have been used in the clinical trials to treat $\operatorname{COVID}-19$ patients $(23,29)$. However, targeting IL-6 signal transduction pathways might be complicated as IL-6 activates both pro-inflammatory pathway through the soluble form of the IL- 6 receptor, and antiinflammatory pathway through the membrane-bound form of the IL-6 receptor $(27,44)$. IL-8 is clearly a pro-inflammatory cytokine that may recruit neutrophils to the infected areas and has been associated with tissue damage. Our studies therefore suggest the possibility of using IL-8 as a biomarker for the disease prognosis of COVID-19 patients and as a potential therapeutic target to treat COVID-19 patients.

\section{CONCLUSION}

Although IL-6 is indicated as a biomarker in predicting severe status of COVID-19 patients, IL-8 is an easily detectible and sensitive biomarker in either mild or severe COVID-19 patients. Serum levels of IL- 8 correlated better than IL- 6 levels with the 
A

Group

. $\begin{array}{ll}\text { High } & \text { Healthy } \\ \text { Low } & \text { Mild Cases } \\ & \text { Severe Cases }\end{array}$

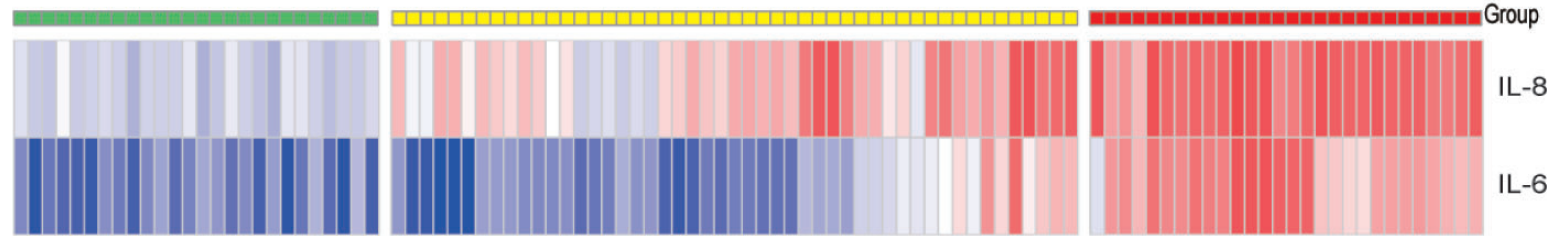

B

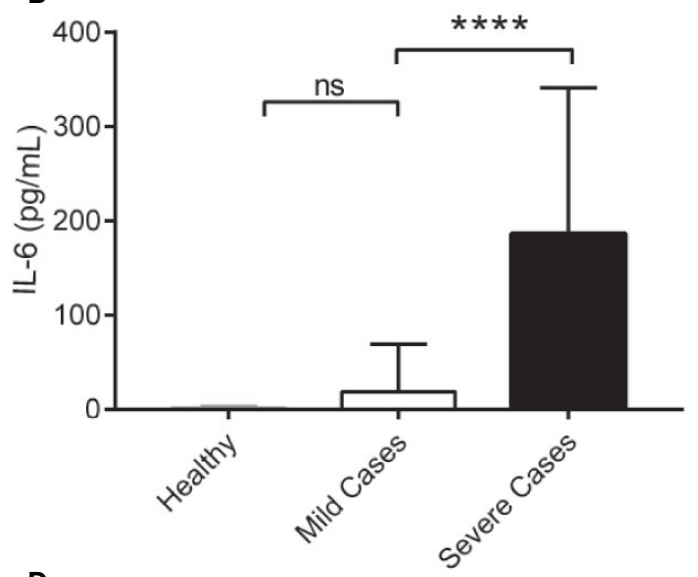

D

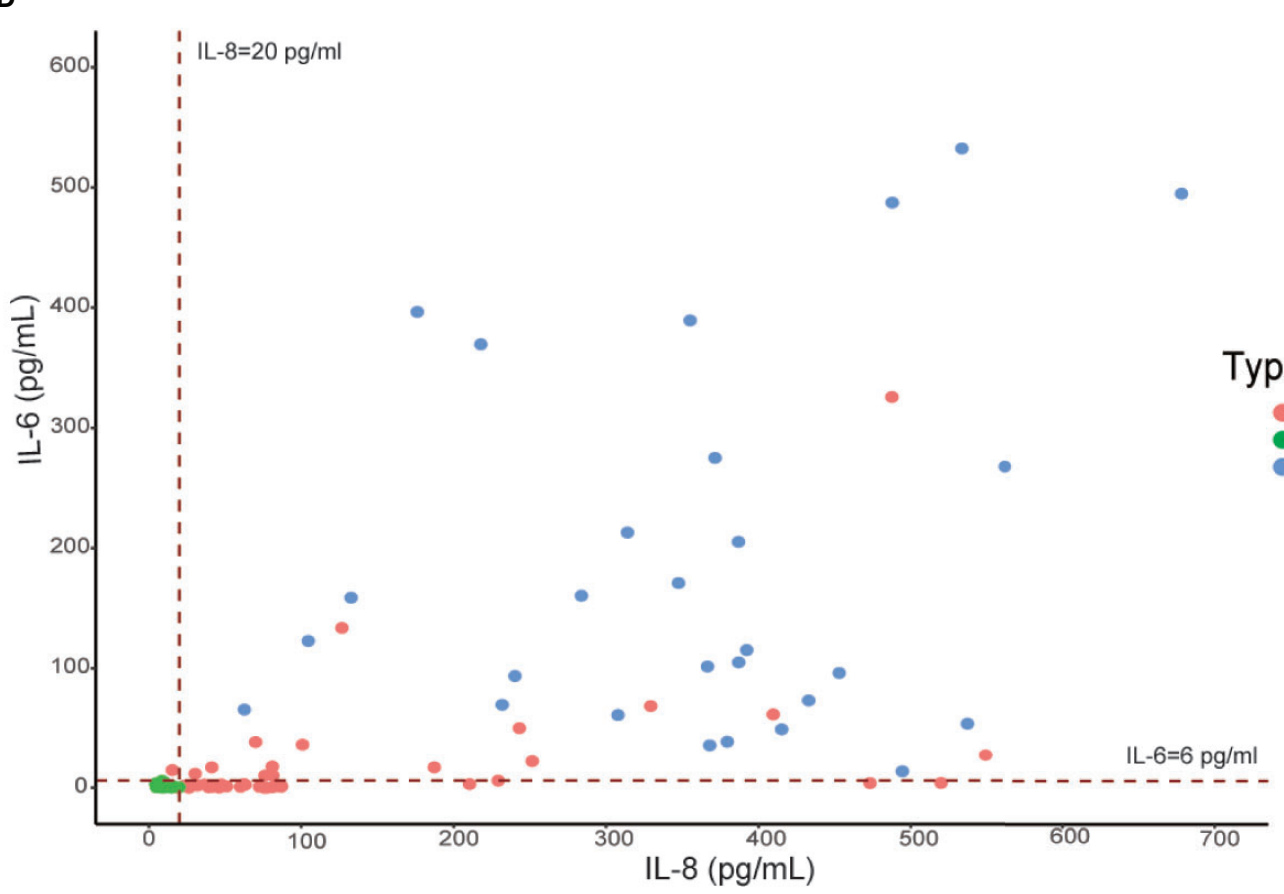

C

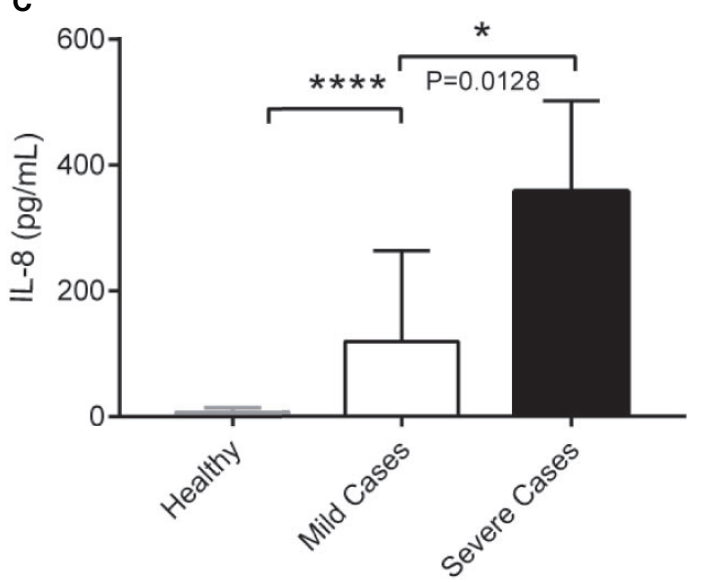

Type

Mild Cases

Healthy

Severe Cases

FIGURE 4 | While IL-6 represents for COVID-19 patients with severe disease conditions, IL-8 is a better indicator of overall COVID-19 diseases. (A) Heatmap of IL-6 and IL-8 levels from COVID-19 patients during different clinical stages. (B, C) The IL-6 (B) and IL-8 (C) levels among different COVID-19 patient groups.

(D) Distribution of IL-6 and IL-8 levels among different COVID-19 patient groups. Dashed lines indicate the lower limit of detection for the ELISA. ELISA data

(B, C) are shown as Mean \pm SEM, ${ }^{*} p<0.05,{ }^{* \star *} p<0.0001$, unpaired Student $t$ test. 
* Clinical Score

$=$ IL-6

$\rightarrow$ IL-8
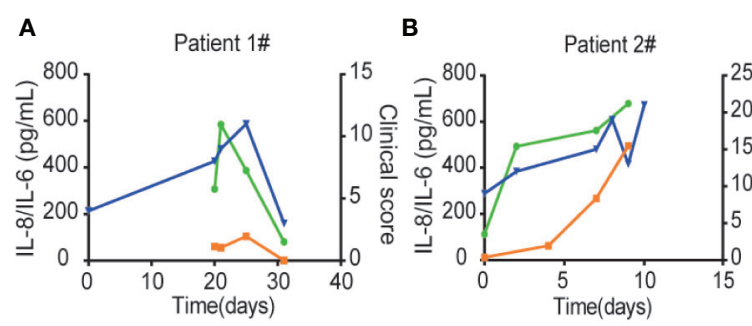

C

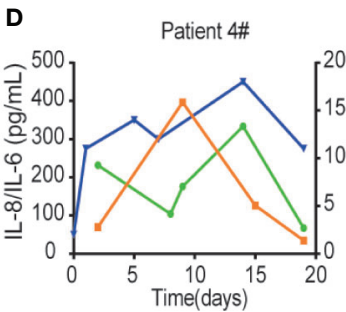

E

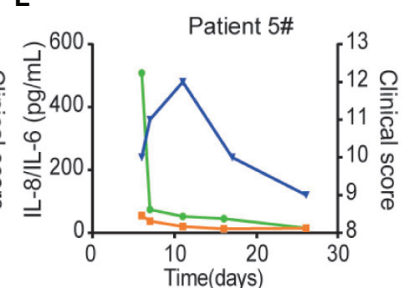

FIGURE 5 | IL-8 may serve as a biomarker to indicate the COVID-19 disease prognosis. (A-E) IL-6 and IL-8 levels were compared with different patients' clinical scores at multiple time points. Left Y-axis displays the concentration of cytokines: IL-6 in orange and IL-8 in green. Right Y-axis displays the clinical scores depicted in blue. X-axis displays the day numbers of COVID-19 diagnosis from the first positive PCR test.

overall clinical disease scores at different stages of the same COVID-19 patients. Thus, IL-8 may act as a biomarker for COVID-19 disease prognosis and target for COVID-19 treatment in the future.

\section{DATA AVAILABILITY STATEMENT}

The original contributions presented in the study are included in the article/supplementary material. Further inquiries can be directed to the corresponding authors.

\section{ETHICS STATEMENT}

The studies involving human participants were reviewed and approved by The Ethics Committee of Taihe hospital (2020KS031). The patients/participants provided their written informed consent to participate in this study. The animal study was reviewed and approved by The Ethics Committee of Taihe hospital (2020KS031). Written informed consent was obtained from the individual(s) for the publication of any potentially identifiable images or data included in this article.

\section{AUTHOR CONTRIBUTIONS}

GC and HY jointly designed this study. YW, JiL, XL, JZ, XX, YJ, and CC performed Quantibody human inflammation array and ELISA assay. LL, MG, MS, and HF collected patient serum samples and clinical data analysis. GC, HY, LL, FM, JK, and
SA wrote and revised the manuscript. All authors contributed to the article and approved the submitted version.

\section{FUNDING}

This project is supported by the Research Funds from Chinese Academy of Medical Sciences (2016-I2M-1-005 and 2019XK310002), National Science funds (2015ZX09102023, NSFC 91542201, 81590765, 31670883, 31870912, 81802870, and 2017YFA0506200), US National Institute of Health funds (AI069120, AI056154, AI140718, and AI028697), the UCLA AIDS Institute and UCLA David Geffen School of Medicine - Eli and Edythe Broad Center of Regenerative Medicine and Stem Cell Research Award Program. HY is supported by Non-profit Central Research Institute Fund of Chinese Academy of Medical Sciences (3332018131), Science funds from Jiangsu Province (BK20170407, BK20151253) and The Innovative and Entrepreneurial Team grant (2018-2021) from Jiangsu Province. LL is supported by Chinese Postdoctoral Science Foundation (2019M650564), CAMS Innovation Fund for Medical Sciences (CIFMS; 2019I2M-1-003) and Innovative and Entrepreneurial Doctor grant (2020-2022) from Jiangsu Province. JiL is supported by Natural Science Foundation of Hubei Province Grant (2016CFB154) and the Scientific and Technological Project of Shiyan City of Hubei Province (18Y26). MS is supported by Key Project of Jiangsu Provincial Health Commission (NO. K2019021).

\section{ACKNOWLEDGMENT}

Grateful acknowledgement is made to all the patients who participated in this study. 


\section{REFERENCES}

1. Matheson NJ, Lehner PJ. How does SARS-CoV-2 cause COVID-19? Science (2020) 369(6503):510-1. doi: 10.1126/science.abc6156

2. Song F, Shi N, Shan F, Zhang Z, Shen J, Lu H, et al. Emerging 2019 Novel Coronavirus (2019-nCoV) Pneumonia. Radiology (2020) 295:210-7. doi: 10.1148/radiol.2020200274

3. Zhou P, Yang XL, Wang XG, Hu B, Zhang L, Zhang W, et al. A pneumonia outbreak associated with a new coronavirus of probable bat origin. Nature (2020) 579(7798):270-3. doi: 10.1038/s41586-020-2012-7

4. Sheahan TP, Frieman MB. The continued epidemic threat of SARS-CoV-2 and implications for the future of global public health. Curr Opin Virol (2020) 40:37-40. doi: 10.1016/j.coviro.2020.05.010

5. Meo SA, Alhowikan AM, Al-Khlaiwi T, Meo IM, Halepoto DM, Iqbal M, et al. Novel coronavirus 2019-nCoV: prevalence, biological and clinical characteristics comparison with SARS-CoV and MERS-CoV. Eur Rev Med Pharmacol Sci (2020) 24:2012-9. doi: 10.26355/eurrev_202002_20379

6. The L. Emerging understandings of 2019-nCoV. Lancet (2020) 395 (10221):311. doi: 10.1016/S0140-6736(20)30186-0

7. Xu X, Chen P, Wang J, Feng J, Zhou H, Li X, et al. Evolution of the novel coronavirus from the ongoing Wuhan outbreak and modeling of its spike protein for risk of human transmission. Sci China Life Sci (2020) 63(3):45760. doi: 10.1007/s11427-020-1637-5

8. Li X, Wang W, Zhao X, Zai J, Zhao Q, Li Y, et al. Transmission dynamics and evolutionary history of 2019-nCoV. J Med Virol (2020) 92(5):501-11. doi: 10.1002/jmv.25701

9. Zaki AM, van Boheemen S, Bestebroer TM, Osterhaus AD, Fouchier RA. Isolation of a novel coronavirus from a man with pneumonia in Saudi Arabia. N Engl J Med (2012) 367(19):1814-20. doi: 10.1056/NEJMoa1211721

10. Drosten C, Gunther S, Preiser W, van der Werf S, Brodt HR, Becker S, et al. Identification of a novel coronavirus in patients with severe acute respiratory syndrome. N Engl J Med (2003) 348(20):1967-76. doi: 10.1056/NEJMoa030747

11. Wu Z, McGoogan JM. Characteristics of and Important Lessons From the Coronavirus Disease 2019 (COVID-19) Outbreak in China: Summary of a Report of 72314 Cases From the Chinese Center for Disease Control and Prevention. JAMA (2020) 323:1239-42. doi: 10.1001/jama.2020.2648

12. Chen N, Zhou M, Dong X, Qu J, Gong F, Han Y, et al. Epidemiological and clinical characteristics of 99 cases of 2019 novel coronavirus pneumonia in Wuhan, China: a descriptive study. Lancet (2020) 395:507-13. doi: 10.1016/ S0140-6736(20)30211-7

13. Ravi N, Cortade DL, Ng E, Wang SX. Diagnostics for SARS-CoV-2 detection: A comprehensive review of the FDA-EUA COVID-19 testing landscape. Biosens Bioelectron (2020) 165:112454. doi: 10.1016/j.bios.2020.112454

14. Qiu X, Xiang Y, Sun J, Wang X, Chen G, Xu X, et al. Dynamic changes of throat swabs RNA and serum antibodies for SARS-CoV-2 and their diagnostic performances in patients with COVID-19. Emerg Microbes Infect (2020) 9 (1):1974-83. doi: 10.1080/22221751.2020.1810133

15. Holborow A, Asad H, Porter L, Tidswell P, Johnston C, Blyth I, et al. The clinical sensitivity of a single SARS-CoV-2 upper respiratory tract RT-PCR test for diagnosing COVID-19 using convalescent antibody as a comparator. Clin Med (Lond) (2020) 20:e209-11. doi: 10.7861/clinmed.2020-0555

16. De Smet K, De Smet D, Ryckaert T, Laridon E, Heremans B, Vandenbulcke R, et al. Diagnostic Performance of Chest CT for SARS-CoV-2 Infection in Individuals with or without COVID-19 Symptoms. Radiology (2020) 202708. doi: 10.1148/radiol.2020202708

17. Kelleni MT. SARS CoV-2 viral load might not be the right predictor of COVID-19 mortality. J Infect (2020) S0163-4453(20)30552-1. doi: 10.1016/ j.jinf.2020.08.018

18. Hagman K, Hedenstierna M, Gille-Johnson P, Hammas B, Grabbe M, Dillner J, et al. SARS-CoV-2 RNA in serum as predictor of severe outcome in COVID-19: a retrospective cohort study. Clin Infect Dis (2020) ciaa1285 ciaa1285. doi: $10.1093 / \mathrm{cid} / \mathrm{ciaa} 1285$

19. Pujadas E, Chaudhry F, McBride R, Richter F, Zhao S, Wajnberg A, et al. SARS-CoV-2 viral load predicts COVID-19 mortality. Lancet Respir Med (2020) 8(9):e70. doi: 10.1016/S2213-2600(20)30354-4

20. Tan C, Li S, Liang Y, Chen M, Liu J. SARS-CoV-2 viremia may predict rapid deterioration of COVID-19 patients. Braz J Infect Dis (2020) 24(6):565-9. doi: $10.1016 /$ j.bjid.2020.08.010
21. Fu Y, Cheng Y, Wu Y. Understanding SARS-CoV-2-Mediated Inflammatory Responses: From Mechanisms to Potential Therapeutic Tools. Virol Sin (2020) 35:266-71. doi: 10.1007/s12250-020-00207-4

22. Huang C, Wang Y, Li X, Ren L, Zhao J, Hu Y, et al. Clinical features of patients infected with 2019 novel coronavirus in Wuhan, China. Lancet (2020) 395:497-506. doi: 10.1016/S0140-6736(20)30183-5

23. Tomasiewicz K, Piekarska A, Stempkowska-Rejek J, Serafinska S, Gawkowska A, Parczewski M. Tocilizumab for patients with severe COVID-19: a retrospective, multi-center study. Expert Rev Anti Infect Ther (2020) 1:1-7. doi: 10.1080/ 14787210.2020 .1800453

24. Wang J, Jiang M, Chen X, Montaner LJ. Cytokine storm and leukocyte changes in mild versus severe SARS-CoV-2 infection: Review of 3939 COVID-19 patients in China and emerging pathogenesis and therapy concepts. J Leukoc Biol (2020) 108:17-41. doi: 10.1002/JLB.3COVR0520-272R

25. Gubernatorova EO, Gorshkova EA, Polinova AI, Drutskaya MS. Drutskaya, IL-6: Relevance for immunopathology of SARS-CoV-2. Cytokine Growth Factor Rev (2020) 53:13-24. doi: 10.1016/j.cytogfr.2020.05.009

26. Weatherhead JE, Clark EH, Vogel TP, Atmar RL, Kulkarni PA. Inflammatory syndromes associated with SARS-CoV-2 infection: dysregulation of the immune response across the age spectrum. J Clin Invest (2020) 130 (12):6194-7. doi: 10.1172/JCI145301

27. Honore PM, Barreto Gutierrez L, Kugener L, Redant S, Attou R, Gallerani A. Inhibiting IL-6 in COVID-19: we are not sure. Crit Care (2020) 24:463. doi: 10.1186/s13054-020-03177-x

28. Maeda T, Obata R, Rizk DD, Kuno T. The Association of Interleukin-6 value, Interleukin inhibitors and Outcomes of Patients with COVID-19 in New York City. J Med Virol (2020). doi: 10.1002/jmv.26365

29. Solis-Garcia Del Pozo J, Galindo MF, Nava E, Jordan J. A systematic review on the efficacy and safety of IL-6 modulatory drugs in the treatment of COVID19 patients. Eur Rev Med Pharmacol Sci (2020) 24:7475-84. doi: 10.26355/ eurrev_202007_21916

30. Amezcua-Guerra LM. Brief annotations on cytokine release syndrome and interleukin-6 therapeutic blockage in SARS-CoV-2/COVID-19. Arch Cardiol Mex (2020) 90(Supl):84-7. doi: 10.24875/ACM.M20000067

31. Palanques-Pastor T, Lopez-Briz E, Poveda Andres JL. Involvement of interleukin 6 in SARS-CoV-2 infection: siltuximab as a therapeutic option against COVID-19. Eur J Hosp Pharm (2020) 27(5):297-8. doi: 10.1136/ ejhpharm-2020-002322

32. Hou H, Zhang B, Huang H, Luo Y, Wu S, Tang G, et al. Using IL-2R/ lymphocytes for predicting the clinical progression of patients with COVID19. Clin Exp Immunol (2020) 201:76-84. doi: 10.1111/cei.13450

33. Wang D, Hu B, Hu C, Zhu F, Liu X, Zhang J, et al. Clinical Characteristics of 138 Hospitalized Patients With 2019 Novel Coronavirus-Infected Pneumonia in Wuhan, China. JAMA (2020) 323(11):1061-9. doi: 10.1001/jama.2020

34. Chakraborty C, Sharma AR, Sharma G, Bhattacharya M, Lee SS. SARS CoV-2 causing pneumonia-associated respiratory disorder (COVID-19): diagnostic and proposed therapeutic options. Eur Rev Med Pharmacol Sci (2020) 24:4016-26. doi: 10.26355/eurrev_202004_20871

35. Lupia T, Scabini S, Mornese Pinna S, Di Perri G, De Rosa FG, Corcione S. novel coronavirus (2019-nCoV) outbreak: A new challenge. J Glob Antimicrob Resist (2020) 21:22-7. doi: 10.1016/j.jgar.2020.02.021

36. Zhu N, Zhang D, Wang W, Li X, Yang B, Song J, et al. China Novel Coronavirus, T. Research, A Novel Coronavirus from Patients with Pneumonia in China, 2019. N Engl J Med (2020) 382:727-33. doi: 10.1056/ NEJMoa2001017

37. Corman VM, Landt O, Kaiser M, Molenkamp R, Meijer A, Chu DK, et al. Detection of 2019 novel coronavirus $(2019-\mathrm{nCoV})$ by real-time RT-PCR. Euro Surveill (2020) 25:3. doi: 10.2807/1560-7917.ES.2020.25.3.2000045

38. Tang EW, Bobenchik AM, Lu S. Testing for SARS-CoV-2 (COVID-19): A General Review. R I Med J (2020) 103(5):20-3. doi: 10.1136/ejhpharm-2020002322

39. Liu Y, Yang Y, Zhang C, Huang F, Wang F, Yuan J, et al. Clinical and biochemical indexes from 2019-nCoV infected patients linked to viral loads and lung injury. Sci China Life Sci (2020) 63:364-74. doi: 10.1007/s11427-020-1643-8

40. Laguna-Goya R, Utrero-Rico A, Talayero P, Lasa-Lazaro M, RamirezFernandez A, Naranjo L, et al. IL-6-based mortality risk model for hospitalized patients with COVID-19. J Allergy Clin Immunol (2020) 20:31027-7. doi: 10.1016/j.jaci.2020.07.009 
41. Luo M, Liu J, Jiang W, Yue S, Liu H, Wei S. IL-6 and CD8+ T cell counts combined are an early predictor of in-hospital mortality of patients with COVID-19. JCI Insight (2020) 5:13.e139024. doi: 10.1172/jci.insight.139024

42. Lipworth B, Chan R, Lipworth S, RuiWen Kuo C. Weathering the Cytokine Storm in Susceptible Patients with Severe SARS-CoV-2 Infection. J Allergy Clin Immunol Pract (2020) 8:1798-801. doi: 10.1016/j.jaip.2020.04.014

43. Prete M, Favoino E, Catacchio G, Racanelli V, Perosa F. SARS-CoV-2 Inflammatory Syndrome. Clinical Features and Rationale for Immunological Treatment. Int J Mol Sci (2020) 21(9):3377. doi: 10.3390/ijms21093377

44. Masia M, Fernandez-Gonzalez M, Padilla S, Ortega P, Garcia JA, Agullo V, et al. Impact of interleukin-6 blockade with tocilizumab on SARS-CoV-2 viral kinetics and antibody responses in patients with COVID-19: A prospective cohort study. EBioMedicine (2020) 60:102999. doi: 10.1016/ j.ebiom.2020.102999
Conflict of Interest: Authors YW and CC were employed by the company Suzhou Func Biotech Inc.

The remaining authors declare that the research was conducted in the absence of any commercial or financial relationships that could be construed as a potential conflict of interest.

Copyright (C) $2021 \mathrm{Li}, \mathrm{Li}$, Gao, Fan, Wang, Xu, Chen, Liu, Kim, Aliyari, Zhang, Jin, Li, $\mathrm{Ma}$, Shi, Cheng and Yang. This is an open-access article distributed under the terms of the Creative Commons Attribution License (CC BY). The use, distribution or reproduction in other forums is permitted, provided the original author(s) and the copyright owner(s) are credited and that the original publication in this journal is cited, in accordance with accepted academic practice. No use, distribution or reproduction is permitted which does not comply with these terms. 